ANNALES

POLONICI MATHEMATICI

$\mathrm{XXX}(\mathbf{1 9 7 5 )}$

\title{
Inéquations portant sur des systèmes linéaires de type parabolique et applications à la recherche de classes d'unicité
}

\author{
par J. Chabrowski (Katowice) et G. Regnaud (Marseille)
}

Résumé. Soit $u$ la solution d'un système linéaire d'inéquations de type parabolique dans un domaine $\Omega \times(0, T)$. On démontre que l'intégrale $\int_{\Omega} \Phi^{2}(t, x) u(t, x)^{2} d x$ est une fonction décroissante de $t$, où $\Phi$ est une fonction poids de la forme

$$
\exp \left\{-m\left[\int_{-1}^{|x|} \frac{d s}{\sqrt{A(s)}}\right]^{2}\right\}
$$

$A$ est une fonction continue, strictement positive définie dans $[-1, \infty)$, telle que la fonction $s \exp \left[-M \int_{-1}^{s} \frac{d t}{\sqrt{A(t)}}\right]$ est bornée pour certain $M$. Comme conséquence on obtient l'unicité du premier problème de Fourier pour les domaines cylindriques non bornés.

Notations et hypothèses. Soient $\Omega$ un ouvert de l'espace euclidien $\boldsymbol{R}^{n}, T$ un réel positif, $S$ le cylindre $\Omega \times[0, T]$. Nous notons $x=\left(x_{1}, \ldots, x_{n}\right)$ un élément de $\boldsymbol{R}^{n},|x|$ la quantité $\sqrt{x_{1}^{2}+\ldots+x_{n}^{2}}$ et $t$ un élément de $[0, T]$.

La boule ouverte de centre l'origine de rayon $\varrho$ dans $\boldsymbol{R}^{n}$ sera notée $B_{e}, S_{e}$ sera' la sphère de centre l'origine, de rayon $\varrho$, dans $\boldsymbol{R}^{n}$.

Nous noterons par: $\omega_{e}$ l'ensemble $B_{e} \cap \Omega, \sigma_{e}$ l'ensemble $S_{Q} \cap \Omega, \Gamma$ la frontière de $\Omega$.

Si $f$ est une fonction différentiable définie dans $S$, nous noterons par $D_{i} f$ la dérivée partielle de la fonction $f$ par rapport à la variable $x_{i}$, $D_{t} f$ la dérivée partielle de la fonction $f$ par rapport à la variable $t$.

Soient $u=\left(u_{1}, \ldots, u_{N}\right)$ et $v=\left(v_{1}, \ldots, v_{N}\right)$ des applications définies dans $S$ à valeur dans $\boldsymbol{R}^{N}$; nous noterons par: $u v$ la fonction définie par $u v=u_{1} v_{1}+\ldots+u_{N} v_{N}, D_{i} u$ l'application définie dans $S$ à valeurs dans $\boldsymbol{R}^{N}$, par $D_{i} u=\left(D_{i} u_{1}, \ldots, D_{i} u_{N}\right), D_{i} u$ l'application définie dans $S$ à valeurs dans $\boldsymbol{R}^{N}$, par $D_{t} u=\left(D_{t} u_{1}, \ldots, D_{t} u_{N}\right)$.

Dans toute la suite, $L$ désignera un opérateur linéaire de type parabolique défini par:

$$
L u \equiv \sum_{i j k} D_{i}\left[\alpha_{i j k}^{p} D_{j} u_{k}\right]-\sum_{k} D_{t} a_{p, k} u_{k}
$$


où $u$ est une application de $S$ dans $\boldsymbol{R}^{N}$ vérifiant certaines propriétés et où les coefficients vérifient les propriétés suivantes:

(a) Jes $a_{i j k}^{p}$ appartiennent à $C^{1}(S)$;

(b) il existe une fonction $F$ appartenant à $C(S)$, telle que, pour tout $\xi=\left(\xi_{i}^{p}\right), \beta=\left(\beta_{i}^{p}\right)$ appartenant à $\boldsymbol{R}^{n \times N}$, on ait:

$$
\sum_{i j k p} \alpha_{i j k}^{p} \xi_{i}^{p} \beta_{j}^{k} \leqslant \lambda F \sum_{i, j j}^{j}\left(\xi_{j}^{p}\right)^{2}+\frac{1}{\lambda} \sum_{i j k p} \alpha_{i j k}^{p} \beta_{i}^{p} \beta_{j}^{k}
$$

et ceci pour tout $\lambda$ réel strictement positif;

(c) $\left|D_{j} a_{i j k}^{p}\right| \leqslant F_{1}$ où $H_{1}^{\prime}$ appartient à $C(S)$;

(d) pour tout $i, j, p, k$, on $a: a_{i j k}^{p}=a_{i j p}^{k}$;

(e) les coefficients $\alpha_{p, k}$ sont localement lipschitziens dans $S$ et vérifient pour tout $\beta=\left(\beta_{p}\right)$ appartenant à $\boldsymbol{R}^{N}$,

$$
0 \leqslant G \beta^{2} \leqslant \sum_{p, k} \alpha_{p, k} \beta_{p} \beta_{k} \leqslant G_{1} \beta^{2} ; \quad \sum_{p k} D_{t}\left(\alpha_{\mu j k}\right) \beta_{p} \beta_{k} \geqslant-H u^{2}
$$

où $G, G_{1}$ et $H$ appartiennent $C(S)$.

Introduction. Dans ce travail, nous avons généralisé une partie des résultats de [3]. Nous introduisons une fonction définie sur $[-1,+\infty[$ que nous noterons toujours par $A$, et qui permettra de généraliser les fonctions poids $\varphi$ qui interviennent dans [3]. Par exemple: dans [3]

$$
\varphi_{m, \beta, \tau}(x, t)=\exp -\frac{m(1+|x|)^{2-\lambda}}{\beta-(t-\tau)},
$$

alors qu'ici nous définissons la fonction poids $\varphi_{A, m, \beta, \varphi}$ par,

$$
\varphi_{A, m, \beta, \tau}(x, t)=\exp \left\{-\frac{m}{\beta-(t-\tau)}\left(\int_{-1}^{|x|} \frac{d u}{\sqrt{A(u)}}\right)^{2}\right\} .
$$

Nous verrons que les résultats obtenus. alors englobent ceux de [3] en choisissant convenablement la fonction $A$. Il nous semble que le fait le plus important est que la fonction $A$ peut posséder des, ,bosses" sans que la classe d'unicité soit changée; exemple: soit l'opérateur:

$$
L u=D_{x}\left(\left[1+x^{2} h(x)\right] D_{x} u\right)-D_{\imath} u
$$

où $h(x)$ est une fonction positive continue vérifiant les propriétés suivantes:

(1) $\max h(x)=1$

(2) pour tout entier $n, h(x)=0$ pour $x$ appartenant à $[n,(n+1) / 2]$. 
Si on applique les résultats de [3], on a un théorème d'unicité pour un tel opérateur dans la classe des fonctions $u$ vérifiant:

$$
u e^{-m_{1} \log x}<K
$$

alors qu'ici nous démontrons qu'il y a unicité pour $u$ vérifiant:

$$
u \exp -m_{1}\left(\int_{-1}^{x} \frac{1}{\sqrt{1+x^{2} h(x)}}\right)^{2}<K
$$

c'est-à-dire une classe beaucoup plus grande à savoir la classe des fonctions $u$ vérifiant

$$
u e^{-2 m_{1} x^{2}}<k \quad\left(m_{1} \text { et } k \text { constantes positives }\right) .
$$

1. DÉfinition 1. Nous dirons que l'application $u$ définie dans $S$ à vảleur dans $\boldsymbol{R}^{N}$ appartient à $\left[C^{1,2}(S)\right]^{N}$ si $u$ appartient à $\left[C^{1}(S)\right]^{N}$ et si pour tout couple $(i, j), D_{i}\left[D_{j} u\right]$ est une application continue dans $\boldsymbol{S}$.

DÉfinition 2. Soit $\psi$ une fonction définie dans $\boldsymbol{R}^{n} \times[0, T]$ à valeurs dans $\boldsymbol{R}_{+}$; on note $\boldsymbol{K}_{\psi}$ l'ensemble des applications $v$ appartenant à $\left[C^{1,2}(S)\right]^{N}$, vérifiant les propriétés suivantes,

(1) $v(x, t)=0$ pour $(x, t)$ appartenant à $\Gamma \times[0, T]$

(2) $\psi v_{p}$ appartient à $L^{2}(S)$ pour tout $p$.

Nous dirons que $v$ est solution du problème $\mathrm{I}$, si $v$ vérifie:

pour tout $r$ supérieur ou égal à $r_{0}\left(r_{0}\right.$ constante positive donnée) et pour tout $t$ appartenant à $[0, T]$

$$
\int_{\omega_{r}}-2 v L v d x \leqslant \int_{\omega_{r}}\left[C_{1} v^{2}+u \sum_{i, j, k, p} \alpha_{i j k}^{p} D_{i} v_{p} D_{j} v_{k}\right] d x
$$

où $C_{1}$ appartient à $C(S)$ et $u$ est une constante positive ou nulle inférieure à 2.

DÉFInition 3. Soit $A$ une fonction continue, strictement positive définie sur le segment $[-1,+\infty[$.

On notera par: $\mathscr{A}$ la fonction définie par

$$
s \rightarrow \mathscr{A}(s)=\int_{-1}^{s} \frac{d u}{\sqrt{A(u)}},
$$

$\psi_{A}$ la fonction définie dans $\boldsymbol{R}^{n} \times[0, T]$ par

$$
(x, t) \rightarrow \psi_{A}(x, t)=\exp -m_{1}[\mathscr{A}(|x|)]^{2},
$$

$\varphi_{A, m, \beta, \tau}$ la fonction définie dans $\boldsymbol{R}^{n} \times[\tau, \tau+\beta / 2]$ par

$$
(x, t) \rightarrow \varphi_{A, m, \beta, \tau}(x, t)=\exp -m \frac{[\mathscr{A}(|y|)]^{2}}{\beta-(t-\tau)}
$$


où $m_{1}, m, \beta$ sont des constantes positives, $\tau$ un réel, et $|y|=\max \left(|x|, r_{0}\right)$ $(\beta<1)$.

Définition 4. Nous dirons que $A$ vérifie l'hypothèse $I\left(B_{\mathrm{T}}\right)$ si sexp $-M_{1}[\mathscr{A}(s)]^{2} \leqslant K_{1}$, pour $s \geqslant 0$, où $M_{1}$ et $K_{1}$ sont constantes positives.

$\Pi$ en résulte que $\int_{-1}^{\infty} \frac{d s}{\sqrt{A(s)}}=\infty$.

Définitron 5. La fonction $A$ étant donnée, nous dirons que les coefficients $H C_{1} G G_{1} F F_{1}$ vórifient l'hypothèse $\mathrm{II}_{A}\left(\mathrm{H}_{\mathrm{U}_{A}}\right)$ si :

$$
\begin{gathered}
G_{1}(x, t) \leqslant K_{1} \exp m_{0}[\mathscr{A}(|x|)]^{2}, \\
\frac{H(x, t)+C_{1}(x, t)}{G(x, t)} \leqslant K_{1}[\mathscr{A}(|x|)]^{2}, \\
\frac{F(x, t)}{G(x, t)} \leqslant K_{1} A(|x|), \\
\frac{F_{1}(x, t)}{G(x, t)} \leqslant K_{1} \exp m_{0}[\mathscr{A}(|x|)]^{2},
\end{gathered}
$$

où $m_{0}$ est une constante positive.

THÉRÈme $\mathrm{I}$. Soit A donnée vérifiant $\mathrm{H}_{\mathrm{I}}$; on suppose que les coefficients $G, G_{1}, F, F_{1}, H, C_{1}$ vérifient $\mathrm{H}_{\mathrm{II}_{A}}$.

Soit $v$ appartenant à $K_{\varphi_{A}}$, $v$ solution du problème $\mathrm{I}$, alors il existe deux constantes positives $\beta_{1}$ et $\beta_{2}$ indépendantes de $\tau$ telles que si $m \leqslant \beta_{1}$ et $m / \beta \geqslant \beta_{2}$, on ait:

$$
\int_{\Omega} \sum_{p, k} \varphi_{A, m, \beta, \tau}^{2} \alpha_{p, k} v_{p} v_{k} d x \text { est une fonction décroissante de } t,
$$
pour $t$ appartenant $\dot{a}[\tau, \tau+\beta / 2] \cap[0, T]$ et ceci pour tout $\tau$,

(2) $\int_{\Omega} \sum_{p, k} \varphi_{A, m, \beta_{,},}^{2}\left(x, \tau_{1}\right) \alpha_{p, k} v_{p} v_{k} d x \quad$ est finie pour tout $\left.\left.t \epsilon\right] 0, T\right]$

$$
\text { et tout } \tau_{1} \text { vérifiant } 0<\tau_{1}-\tau<\beta / 2,
$$

(3) $\varphi_{A, m, \beta, \tau}^{2}\left(x, \tau_{1}\right) \sum_{i, j, k, p} a_{i j k}^{p} D_{i} v_{p} D_{j} v_{k} \quad$ appartient $\grave{a} L^{1}\left(\Omega \times\left[\tau_{2}, T\right]\right)$

$$
\text { où } \tau_{2}>0 \text { et } 0<\tau_{1}-\tau<\beta / 2 .
$$

Démonstration. La démonstration de ce théorème se fera en quatre étapes:

Etape 1. Nous rappelons un résultat intermédiaire obtenu dans [3]. Soit $\varphi$ une fonction définie dans $\boldsymbol{R}_{+} \times\left[t_{1}, t_{2}\right] \grave{\mathrm{a}}$ valeurs dans $\boldsymbol{R}_{+}$, où $t_{1}, t_{2}$ 
vérifient $0 \leqslant t_{1}<t_{2} \leqslant T$, vérifiant,

(1) $\varphi$ est lipschitzienne dans tout borné de $\boldsymbol{R}_{+} \times\left[t_{1}, t_{2}\right]$,

(2) $\varphi(|x|, t)=\varphi\left(r_{0}, t\right)$ pour tout $|x| \leqslant r_{0}$,

(3) les fonctions $t \rightarrow \varphi(|x|, t)$ et $|x| \rightarrow \varphi(|x|, t)$ sont décroissantes,

(4) $\varphi$ est solution presque partout de l'inégalité suivante,

$$
-\varphi \varphi_{t} G-\varphi^{2}\left[H+C_{1}\right]-\frac{32}{2-\mu} F_{|x|}^{2} \geqslant 0
$$

où $\varphi_{|x|}$ représente la dérivée partielle de la fonction $\varphi$ par rapport à la variable $|x|$, et $\varphi_{t}$ la dérivée partielle par rapport à la variable $t$.

Si $v$ est solution du problème $\mathrm{I}$, alors $v$ vérifie l'inégalité suivante:

$$
\begin{aligned}
\int_{\tau_{1}} \int_{\omega_{r}}^{\tau_{2}} & {\left[-\varphi \varphi_{t} G v^{2}+\frac{2-\mu}{2} \varphi^{2} \sum_{i j k p} a_{i, j, k}^{p} D_{j} v_{p} D_{j} v_{k}\right] d x d t } \\
\leqslant \int_{\tau_{1}}^{\tau_{2}} \int_{\sigma_{r}} \sum_{i j k p} 2 \varphi^{2} v_{p} a_{i, j, k}^{p} D_{j} v_{k} \cdot \frac{x_{j}}{|x|} d s d t+ & {\left[\int_{\omega_{r}} \sum_{p k} \varphi^{2} a_{p, k} v_{p} v_{k} d x\right]_{\ell=\tau_{1}}-} \\
& -\left[\int_{\omega_{r}} \sum_{p, k} \varphi^{2} a_{p, k} v_{p} v_{k} d x\right]_{i=\tau_{2}}
\end{aligned}
$$

où $\left(\tau_{1}, \tau_{2}\right)$ vérifie $t_{1} \leqslant \tau_{1}<\tau_{2} \leqslant t_{2}$. On démontre ce résultat en considérant l'identité:

$$
-2 \varphi^{2} v L v \equiv-\sum_{i, j, k, p} 2 \varphi^{2} v D_{i}\left[a_{i j k}^{p} D_{j} v_{k}\right]+\sum_{p k} \varphi^{2} v D_{t} a_{p k} v_{k}
$$

On obtient par des calculs élémentaires,

$$
\begin{aligned}
& -2 \varphi^{2} v L v \geqslant-\sum_{i j k p} D_{i}\left[2 \varphi^{2} v_{p} \alpha_{i j k}^{p} D_{i} v_{k}\right]+\sum_{x k} D_{t}\left[\varphi^{2} \alpha_{p k} v_{p} v_{k}\right]+ \\
& +\left[-2 \varphi \varphi_{t} G-\varphi^{2} H-4 \lambda \varphi_{|x|}^{2} F^{2}\right] v^{2}+2 \varphi^{2}\left[1-\frac{2}{\lambda}-\frac{\mu}{2}\right] \sum_{i j k p} \alpha_{i j k}^{p} D_{i} v_{p} D_{j} v_{k},
\end{aligned}
$$

où $\lambda$ est un réel positif; si on prend $\lambda=8 / 2-\mu$, et si on intégre l'inégalité précédente sur $\omega_{r} \times\left[\tau_{1}, \tau_{2}\right], t_{1} \leqslant \tau_{1}<\tau_{2} \leqslant t_{2}$ et, en utilisant la méthode énoncée dans [3] nous obtenons le résultat.

Eatpe 2. Montrons que $\varphi=\varphi_{A, m, \beta, \tau}$ vérifient les conditions demandées précédemment, pourvu que $m$ et $\beta$ vérifient $m \leqslant 2-\mu / 256 K_{1}, m / \beta \geqslant 2 K_{1}$. En éffet 1, 2, 3, sont bien vérifiées, il reste à vérifier (4).

Remplaçons $\varphi$ par $\varphi_{A, m, \beta, \tau}$ dans le premier membre de (4): si $|x|<r_{0}$, on a, en utilisant l'hypothèse $\mathrm{II}_{A}$,

$$
\frac{m\left[\mathscr{A}\left(r_{0}\right)\right]^{2}}{[\beta-(t-\tau)]^{2}}-K_{1}[\mathscr{A}(|x|)] \geqslant\left[\frac{m}{\beta^{2}}-K_{1}\right]\left[\mathscr{A}\left(r_{0}\right)\right]^{2},
$$


quantité qui est positive si $m / \beta \geqslant K_{1}$ (par hypothese $\beta<1$ ); si $|x|>r_{0}$, on obtient,

$$
\left[\frac{m}{[\beta-(t-\tau)]^{2}}-K_{1}-\frac{128 m^{2} K_{1}}{(2-\mu)[\beta-(t-\tau)]^{2}}\right][\mathscr{A}(r)]^{2}
$$

quantité qui est positive si $m / \beta \geqslant 2 K_{1}$ et $m<2-\mu / 256 K_{1}$.

Done si $m / \beta \geqslant 2 K_{1}$ et $m<2-\mu / 256 K_{1}, \varphi_{A, m, \beta, \tau}$ vérifje (1), (2), (3), (4), donc (5) est verifiée si on remplace $\varphi$ par $\varphi_{\dot{A}, m, \beta, \tau}$.

Etape 3. Montrons que si $v$ appartient à $K_{\psi_{A}}$, si $m / \beta \geqslant \max \left(3 M_{1}+m_{1}\right.$, $\left.M_{1}+2 m_{0}+m_{1}, m_{0}+3 M_{1}+m_{1}\right)$, alors $\varphi_{A, m, \beta_{,},}$et $v$ vérifient:

(6) Pour tout $\left(\tau_{1}, \tau_{2}\right)\left(\tau \leqslant \tau_{1}<\tau_{2} \leqslant \tau+\beta / 2\right)$, il existe une suite $r_{m}$ tendant vers l'infini et une suite $R_{m}$ tendant vers zéro, telles que,

$$
\int_{\tau_{2}}^{\tau_{1}} \int_{\sigma_{\tau_{m}}} \sum_{i j k p} 2 \varphi_{A, m, \theta, \tau}^{2} \frac{x_{i}}{|x|} a_{i j k}^{p} v_{p} D_{j} v_{k} d S d t \leqslant R_{m} .
$$

Pour démontrer ce résultat considérons l'intégrale,

$$
I(r)=\int_{\tau_{1}}^{\tau_{2}} \int_{\omega_{r}} \sum_{i j k p} 2 \varphi_{A, m, B, \tau}^{2} x_{i} \alpha_{i j k}^{p} v_{p} D_{j} v_{k} d x d t,
$$

où $\tau_{1}, \tau_{2}$ vérifient $\tau \leqslant \tau_{1}<\tau_{2} \leqslant \tau+\beta / 2, r>r_{0}$.

On a:

$$
\begin{aligned}
& I(r)=\int_{\tau_{1}} \int_{\omega_{r}} \sum_{i j k p} D_{j}\left[\varphi_{A, m, \beta, \tau}^{2} x_{i} a_{i j k}^{p} v_{p} v_{k}\right] d x d t- \\
& -\int_{\tau_{2}}^{\tau_{2}} \int_{\omega_{r}} \sum_{i j k p}\left[\delta_{i j}-\frac{4 m}{\beta-(t-\tau)} \mathscr{A}(|x|) \frac{1}{\sqrt{A(|x|)}} \frac{x_{i} x_{j}}{|x|}\right] \varphi_{A, m, \beta, \tau}^{2} a_{i j k}^{p} v_{p} v_{k} d x d t- \\
& -\int_{\tau_{1}} \int_{\omega_{r}} \sum_{i j k p} \varphi_{A, m, \beta, \tau}^{2} x_{i} v_{p} v_{k} D_{j} a_{i j k}^{p} d x d t=B_{1}+B_{2}+B_{3},
\end{aligned}
$$

où $\delta_{i j}$ est le symbole de Kronecker.

Etudions chaque terme du deuxieme membre de l'égalité précédente. Considerons $B_{2}$;

Si $\sqrt{A(x)}>n^{2} \frac{8 m}{\beta} \mathscr{A}(|x|) \cdot|x|$ en un point de $\boldsymbol{R}^{n} \times\left[\tau_{1}, \tau_{2}\right]$, alors nous avons:

$$
\begin{aligned}
\sum_{i j k p}\left[\delta_{i j}-\frac{4 m}{\beta-(t-\tau)} \mathscr{A}(|x|) \frac{1}{\sqrt{A(x)}} \frac{x_{i} x_{j}}{|x|}\right] \alpha_{i j k}^{p} v_{p} v_{k} \\
\geqslant \sum_{i j k p}\left[\delta_{i j}-\frac{1}{n^{2}} \frac{x_{i} x_{j}}{|x|^{2}}\right] \alpha_{i j k}^{p} v_{p} v_{k} .
\end{aligned}
$$


Introduisons les formes quadratiques

$$
\begin{aligned}
& \mathscr{F}_{1}=\sum_{i j} d_{i j} \lambda_{i} \lambda_{j} \quad \text { où } d_{i j}=\sum_{p k} a_{i j k}^{p} v_{p} v_{k}, \\
& \mathscr{F}_{2}=\sum_{i j}\left[\delta_{i j}-\frac{1}{n^{2}} \frac{x_{i} x_{j}}{|x|^{2}}\right] \mu_{i} \mu_{j} .
\end{aligned}
$$

En vertu de l'hypothèse (b), la forme $\mathscr{F}_{1}$ est positive. De plus,

$$
\mathscr{F}_{2}=|\mu|^{2}-\sum_{i j} \frac{1}{n^{2}} \frac{x_{i} x_{j}}{|x|^{2}} \mu_{i} \mu_{j} \geqslant 0
$$

est aussi une forme quadratique positive. Alors, il est bien connu que:

$$
\begin{gathered}
\sum_{i j} d_{i j}\left[\delta_{i j}-\frac{1}{n^{2}} \frac{x_{i} x_{j}}{|x|^{2}}\right] \geqslant 0, \quad \text { c'est-à-dire, } \\
\sum_{i j k p}\left[\delta_{i j}-\frac{1}{n^{2}} \frac{x_{i} x_{j}}{|x|^{2}}\right] \alpha_{i j k}^{p} v_{p} v_{k} \geqslant 0
\end{gathered}
$$

Si $\sqrt{A(x)} \leqslant n^{2} \frac{8 m}{\beta} \mathscr{A}(|x|) \cdot|x|$, en un point de $\boldsymbol{R}^{n} \times\left[\tau_{1}, \tau_{2}\right]$, alors nous avons:

$$
\begin{aligned}
-\sum_{i j k p}\left[\delta_{i j}-\frac{4 m}{\beta-(t-\tau)} \mathscr{A}(|x|) \frac{1}{\sqrt{A(|x|)}} \frac{x_{i} x_{j}}{|x|}\right] \alpha_{i j k}^{p} v_{p} v_{k} \\
\leqslant \frac{32 m}{\beta} K_{1}^{2} \mathscr{A}(|x|) \sqrt{\mathcal{A}(|x|)} \exp \left[m_{0}[\mathscr{A}(|x|)]^{2}\right] \cdot|x||v|^{2} \\
\leqslant \frac{256}{\beta^{2}} m^{2} n^{2} K_{1}^{2}[\mathscr{A}(|x|)]^{2}|x|^{2}|v|^{2} \exp m_{0}[\mathscr{A}(|x|)]^{2}
\end{aligned}
$$

Donc, dans tous les cas, nous avons:

$$
B_{2} \leqslant \frac{256}{\beta^{2}} m^{2} n^{2} K_{1}^{2} \int_{\tau_{\mathrm{J}}}^{\tau_{2}} \int_{\alpha_{r}}[\mathscr{A}(|x|)]^{2}|x|^{2} \varphi_{A, m, \beta, \tau}^{2} v^{2} \exp m_{0}[\mathscr{A}(|x|)]^{2} d x d t .
$$

En utilisant l'hypothèse $H_{1}$, nous avons:

$$
\begin{aligned}
B_{2} \leqslant & \frac{256}{\beta^{2}} m^{2} n^{2} K_{1}^{2} \int_{\tau_{1}}^{\tau_{2}} \int_{\omega_{r}}[\mathscr{A}(|x|)]^{2} \exp -M_{1}[\mathscr{A}(|x|)]^{2} \cdot|x|^{2} \times \\
& \times \exp -2 M_{1}[\mathscr{A}(|x|)]^{2} \cdot \exp \left(-\frac{2 m}{\beta-(t-\tau)}+3 M_{1}\right)[\mathscr{A}(|x|)]^{2} \cdot v^{2} d x d t .
\end{aligned}
$$


Remarquons que si $\mathrm{H}_{1}$ est vraie, $[\mathscr{A}(|x|)]^{2} \exp -M_{1}[\mathscr{A}(|x|)]^{2}$ tend vers zéro quand $|x|$ tend vers l'infini et que $|x|^{2} \exp -2 M_{1}[\mathscr{A}(|x|)]^{2} \leqslant K_{1}^{2}$; comme $v$ appartient ̀̀ $K_{\varphi_{A}}$, si $m / \beta \geqslant 3 M_{1}+m_{1}$, nous aurons $B_{2} \leqslant R_{1}, R_{1}$ constante positive.

Considérons $B_{3}$,

$$
\begin{array}{r}
B_{3} \leqslant \int_{\tau_{1}}^{\tau_{2}} \int_{\omega_{r}} K_{1}^{2} n^{2} N^{2}|x| \exp \left\{-M_{1}[\mathscr{A}(|x|)]^{2}\right\} \\
\exp \left\{\left[-\frac{2 m}{\beta-(t-\tau)}+M_{1}+\right.\right. \\
\left.\left.+2 m_{0}\right][\mathscr{A}(|x|)]^{2}\right\} v^{2} d x d t
\end{array}
$$

Donc si $m / \beta \geqslant M_{1}+2 m_{0}+m_{1}$, nous avons:

$$
B_{3} \leqslant R_{2} R_{2} \quad \text { constante positive. }
$$

Nous avons donc, si $m / \beta \geqslant \max \left(3 M_{1}+m_{1}, M_{1}+2 m_{0}+m_{1}\right)$,

$$
I(r) \leqslant \int_{\tau_{1}}^{\tau_{2}} \int_{\sigma_{r}} \sum_{i j k p} \varphi_{A, m, \beta, \tau}^{2} a_{i j k}^{p} \frac{x_{i} x_{j}}{|x|} v_{p} v_{k} d s d t+R_{1}+R_{2},
$$

car d'après les hypothèses faites, nous pouvons appliquer le lemme $\mathbf{A}$ (voir §5).

Considérons l'intégrale

$$
B_{1}=\int_{\tau_{1}}^{\tau_{2}} \int_{\sigma_{r}} \sum_{i j k p} \varphi_{A, m, \beta, \tau}^{2} a_{i j k}^{p} \frac{x_{i} x_{j}}{|x|} v_{p} v_{k} d s d t
$$

nous avons,

$$
\begin{aligned}
B_{1} & \leqslant \int_{\tau_{1}}^{\tau_{2}} \int_{\sigma_{\tau}} 4 K_{1}^{2} A(r)|x| \exp \left\{\left[-\frac{2 m}{\beta-(t-\tau)}+m_{0}\right][\mathscr{A}(|x|)]^{2}\right\} v^{2} d s d t \\
& \leqslant 4 K_{1}^{2} \exp \left\{\left[-\frac{2 m}{\beta}+m_{0}\right][\mathscr{A}(r)]^{2}\right\} r A(r) \int_{\tau_{1}}^{\tau_{2}} \int_{\sigma_{r}} v^{2} d s d t=J(r) .
\end{aligned}
$$

Soit $r_{1}>0$ donné et supposons que pour tout $r \geqslant r_{1}, J(r) \geqslant R_{3}\left(R_{3}\right.$ constante positive donnée) nous avons donc, pour tout $r \geqslant r_{1}$ :

d'où :

$$
\begin{aligned}
& \frac{R_{3}}{A(r)} \leqslant 4 r K_{1}^{2} \exp \left\{-M_{1}(\mathscr{A}(r))^{2}\right\} \cdot \exp \left\{\left(-\frac{2 m}{\beta}+\right.\right. \\
&\left.\left.+m_{0}+M_{1}\right)[\mathscr{A}(r)]^{2}\right\} \int_{\tau_{1}}^{\tau_{2}} \int_{\sigma_{r}} v^{2} d s d t,
\end{aligned}
$$

$$
\frac{\sqrt{R_{3}}}{\sqrt{A(r)}} \leqslant 2 K_{1}^{2} \exp \left\{\frac{1}{2}\left(-\frac{2 m}{\beta}+m_{0}+M_{1}\right)(\mathscr{A}(r))^{2}\right\} \sqrt{\int_{\tau_{1}}^{\tau_{2}} \int_{\sigma_{r}} v^{2} d s d t .}
$$


Intégrons cette inégalité sur $\left[r_{1}, \varrho\right]\left(\varrho \geqslant r_{1}\right)$ et utilisons l'inégalitê de Hölder, nous avons,

$$
\begin{aligned}
\int_{r_{1}}^{e} \frac{\sqrt{R_{3}}}{\sqrt{A(s)}} d s & \leqslant 2 K_{1}^{2}\left[\int_{r_{1}}^{e} \exp \left\{-2 M_{1}[\mathscr{A}(s)]^{2}\right\} d s\right]^{1 / 2} \times \\
\times & {\left[\int_{r_{1}}^{e}\left(\exp \left\{\left(-\frac{2 m}{\beta}+m_{0}+3 M_{1}\right)[\mathscr{A}(r)]^{2}\right\} \int_{\tau_{1}}^{\tau_{2}} \int_{\sigma_{r}} v^{2} d s d t\right) d r\right]^{1 / 2} . }
\end{aligned}
$$

Si on suppose que $m / \beta \geqslant m_{0}+3 M_{1}+m_{1}$, en utilisant $\mathrm{H}_{1}$ et le fait que $v$ appartient à $K_{\psi_{A}}$, nous avons:

$$
\int_{r_{1}}^{e} \frac{\sqrt{R_{3}}}{\sqrt{A(s)}} d s \leqslant 2 K_{1}^{3}\left(\int_{\tau_{1}}^{\varrho} \frac{1}{\varrho^{2}} d \varrho\right)^{1 / 2}\left[\int_{\tau_{1}}^{\tau_{2}} \int_{\omega_{\varrho}} \exp \left\{-m_{1}[\mathscr{A}(|x|)]^{2}\right\} v^{2} d x d t\right]^{1 / 2} \leqslant R_{4},
$$

où $R_{4}$ est une constante positive indépendante de $\varrho$; ce qui est impossible, car quand $\varrho$ tend vers l'infini, le premier membre de l'inégalité tend vers l'infini.

Donc il existe une suite $r_{m}$ tendant vers l'infini, telle que si $m / \beta$ $\geqslant \max \left(3 M_{1}+m_{1}, \quad M_{1}+2 m_{0}+m_{1}, \quad m_{0}+3 M_{1}+m_{1}\right)$, alors nous avons, $I\left(r_{m}\right) \leqslant R$ ( $R$ constante positive indépendante de $m$ ).

Montrons que pour tout $r_{m}$, il existe $r_{m}^{\prime} \geqslant r_{m}$ tel que

$$
I_{1}\left(r_{m}^{\prime}\right)=\int_{\tau_{1}}^{\tau_{2}} \int_{\sigma_{\tau^{\prime}}} \sum_{i, j, k, p} 2 \varphi_{A, m, \beta, \tau}^{2} x_{i} a_{i j k}^{p} v_{p} D_{j} v_{k} d s d t \leqslant R
$$

En effet, si ceci était faux, alors, pour tout $r_{p}>r_{m}$, on aurait, $I\left(r_{p}\right)>I\left(r_{m}\right)+\left(r_{p}-r_{m}\right) R$ qui tendrait vers l'infini quand $r_{p}$ tendrait vers l'infini, ce qui est impossible.

Ce qui démontre (6) (la suite $r_{m}$ de (6) est égale à $r_{m}^{\prime}$ et $R_{m}=R / r_{m}^{\prime}$ ).

Etape 4. Supposons que:

$$
\frac{m}{\beta} \geqslant \max \left[2 K_{1}, 3 M_{1}+m_{1}, M_{1}+2 m_{0}+m_{1}, m_{0}+3 M_{1}+m_{1}\right]=\beta_{2},
$$

et

$$
m \leqslant \frac{2-\mu}{256 K_{1}}=\beta_{1} .
$$

Alors nous pouvons utiliser l'inégalité (5) dans laquelle nous posons $r=r_{m}$ et faire tendre $m$ vers l'infini. Les résultats du théorème I découlent immédiatement de cette inégalité (voir aussi [3], thèoréme 2.1.1).

2. Cas $N=1$. Nous dirons que $v$ est solution du problème II, si $v$ vérifie: $-2 v L v \leqslant C_{1} v^{2}+\mu \sum_{i j} \alpha_{i i} D_{i} v D_{j} v$, pour tout $(x, t)$ appartenant à 
$\Omega \times[0, \tau]$ où $C_{1}$ appartient à $C(S)$ et $\mu$ est une constante positive ou nulle, inférieure à 2 .

DÉfinimion 6. Soit $v$ une fonction définie dans $S$ à valeurs dans $\boldsymbol{R}$, nous définissons deux nouvelles fonctions $v_{+}$et $v_{-}$par:

$$
\begin{aligned}
& v_{+}(x, t)=\max [0, v(x, t)], \\
& v_{-}(x, t)=\max [0,-v(x, t)] .
\end{aligned}
$$

DÉfinition 7. Soit $\psi$ une fonction définie dans $\boldsymbol{R}^{n} \times[0, T]$ à valeurs dans $\boldsymbol{R}_{+}$, on note $K_{+\varphi}$ (respectivement $K_{-\psi}$ ) l'ensemble des applications appartenant à $C^{1,2}(S)$ vérifiant les propriétés suivantes:

(1) $v_{+}(x, t)=0$ (respectivement $v_{-}(x, t)=0$ ) pour $(x, t)$ appartenant $\Gamma \times[0, T]$,

(2) $\psi v_{+}$(respectivement $\psi v_{-}$) appartient à $L^{2}(S)$.

THÉoRغ̀me II. Soient: $A$ donnée vérifiant $\mathrm{H}_{1}$, on suppose que les coefficients $H, C_{1}, G, G_{1}, F, F_{1}$ vérifiant $\mathrm{H}_{\mathrm{II}_{A}}, v$ appartenant $\dot{a} K_{+\psi_{A}}, v$ solution du problème II, alors il existe deux constantes positives $\beta_{1}$ et $\beta_{2}$ indépendantes de $\tau$, telles que si $m \leqslant \beta_{1}$ et $m / \beta \geqslant \beta_{2}$, on ait:

(1) $\int_{\Omega} \varphi_{A, m, \beta, \tau}^{2} \alpha v_{+}^{2} d x$ fonction décroissante de $t$, pour $t$ appartenant $\grave{a}$ $\left[\tau, \tau+\frac{\beta}{2}\right] \cap[0, T]$ et ceci pour tout $\tau$,

(2) $\int \varphi_{A, m, \beta, \tau}^{2}\left(x, \tau_{1}\right) a v_{+}^{2} d x$ finie pour tout $\left.\left.t \epsilon\right] 0, T\right]$ et tout $\tau_{1}$ vérifiant $0<\tau_{1}-\tau<\beta / 2$,

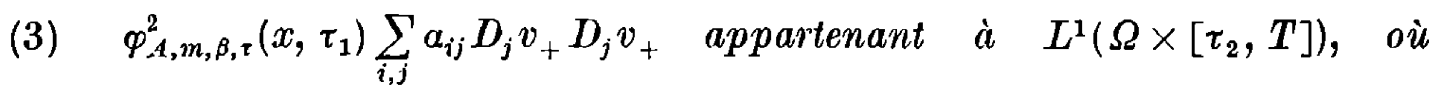
$\tau_{2}>0$ et $0<\tau_{1}-\tau<\beta / 2$.

Démonstratiou. A l'aide du lemme $B, \S 5$, nous avons un résultat intermédiaire qui remplace l'étapel dans la démonstration du théorème 1 , à savoir: si $\varphi$ vérifie $1-2-3-4$, si $v$ est solution du problème II, alors $v_{+}$ (respectivement $v_{-}$) vórifie l'inégalité suivante:

$$
\begin{aligned}
& \left(5^{\prime}\right) \int_{\tau_{1}}^{\tau_{2}} \int_{\omega_{r}}\left[-\varphi \varphi_{i} G v_{+}^{2}+\frac{2-\mu}{2} \varphi^{2} \sum_{i j} \alpha_{i j} D_{i} v_{+} D_{j} v_{+}\right] d x d t \\
& \leqslant \int_{\tau_{1}}^{\tau_{2}} \int_{\sigma_{r}} \sum_{i j} 2 \varphi^{2} v_{+} a_{i j} \frac{x_{i}}{|x|} D_{j} v d s d t+\left[\int_{\omega_{r}} \varphi^{2} a v_{+}^{2} d x\right]_{l=\tau_{1}}-\left[\int_{\omega_{r}} \varphi^{2} a v_{+}^{2} d x\right]_{t=\tau_{2}}
\end{aligned}
$$

qui remplace l'inégalité (5). La suite de la démonstration est identique à la démonstration précédente, sauf que la propriété (6) sera remplacée par $\left(6^{\prime}\right)$ 
(6) pour tout $\tau_{1}, \tau_{2}\left(\tau \leqslant \tau_{1}<\tau_{2} \leqslant \tau+\beta / 2\right)$, il existe une suite $r_{m}$ tendant vers l'infini et une suite $\boldsymbol{R}_{m}$ tendant vers zéro, telles que,

$$
\int_{\tau_{1}}^{\tau_{2}} \int_{\sigma_{r_{m}}} \sum_{i j} 2 \varphi^{2} v_{+} a_{i j} \frac{x_{i}}{|x|} D_{j} v d s d t \leqslant R_{m},
$$

qui se démontre d'une manière analogue à (6).

\section{Applications.}

ThÉonèmes D'UNiciré. Considérons le système du type parabolique suivant:

$$
L_{1} v \equiv I v+\sum_{i k} b_{i k}^{p} D_{i} v_{k}+c_{k}^{p} v_{k} \equiv L v+B v+C v .
$$

On dira que $v$ est solution du problème III, si

$$
\begin{aligned}
v(x, 0) & =0, \\
v(x, t) & =0 \\
L_{1} v & =0 .
\end{aligned} \text { pour }(x, t) \text { appartenant d̀ } i \times[0, T] .
$$

НуротнЀsE III. Les $b_{i k}^{p}$ et $c_{k}^{p}$ appartiennent à $C(S)$ et vérifient: pour tout $r \geqslant r_{0}\left(r_{0}\right.$ constante positive fixée), pour tout $t$ appartenant $\dot{a}[0, T]$,

$$
\int_{\omega_{r}} 2 v[B v+C v] d x \leqslant \int_{\omega_{r}}\left[C_{1} v^{2}+\mu \sum_{i j k p} a_{i j k}^{p} D_{i} v_{p} D_{j} v_{k}\right] d x
$$

où $C_{1}$ appartient à $C(S)$ et $\mu$ est une constante positive ou nulle inférieure à 2 .

ThÉORÈme III. Soient: $A$ donnée vérifiant $\mathrm{H}_{1}$, les coefficients $H, C_{1}$, $F, F_{1}, G, G_{1}$ vérifiant $\mathrm{H}_{\mathrm{II}_{A}}$ et $B$ et $C$ vérifiant $\mathrm{H}_{\mathrm{III}}$, vappartenant $\dot{a} K_{\psi_{A}}$, $v$ solution $d u$ problème III, alors $v$ est identiquement nulle dans $\mathbf{S}$.

La démonstration est identique à celle du théorème 3.1.1 de [3]. Cas $N=1$.

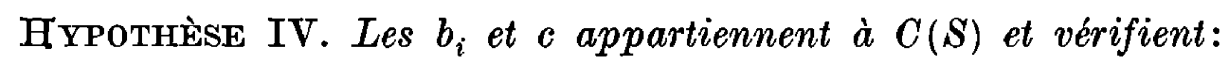

$$
2 v(B v+C v) \leqslant C_{1} v^{2}+\mu \sum_{i j} a_{i j} D_{i} v D_{j} v
$$

ou $C_{1}$ appartient à $C(S)$ et $\mu$ est une constante positive ou nulle inférieure à 2 .

THÉoRÈme IV (principe de maximum). Soient: $A$ donnée vérifiant $\mathbf{H}_{1}$, les coefficiants $b_{i}$ et c vérifiant l'hypothèse IV, les coefficients $H, C_{1}, F, F_{1}$, $G, G_{1}$ verifiant $\mathrm{H}_{\mathrm{Ir}_{A}}$, soient $u_{1}$ et $u_{2}$ appartenant $\grave{a} K_{\psi_{A}}$, solutions de l'équation $L_{1} u=0, s i$

$$
\begin{aligned}
& u_{1}(x, t) \leqslant u_{2}(x, t) \quad \text { pour }(x, t) \text { appartenant } \dot{a} \Gamma \times[0, T] \text { et } \\
& u_{1}(x, 0) \leqslant u_{2}(x, 0) \quad \text { pour } x \text { appartenant } \grave{a} \Omega,
\end{aligned}
$$

alors $u_{1}(x, t) \leqslant u_{2}(x, t)$ pour $(x, t)$ appartenant $\dot{a} S$. 
Démonstration. Si nous posons $v(x, t)=u_{1}(x, t)-u_{2}(x, t)$, alors $v_{+}(x, 0)=0$ et $v_{+}(x, t)=0$ pour $(x, t)$ appartenant à $\Gamma \times[0, T]$. I suffit d'appliquer le théorème II pour démontrer ce théorème.

4. Résultat optimaux. Nous démontrons ici que dans un certain sens il n'est pas possible d'améliorer les résultats précédențs. Nous donnons une solution du problème III, non identiquement nulle, où les coéfficients $H, C_{1}, F, F_{1}, G, G_{1}$ vérifient $\mathrm{H}_{\mathrm{II}_{A}}$, la fonction $u$ solution du problème $I I$, vérifie:

$u$ appartient à̀ $K_{\psi_{A, \varepsilon}}$, où $\psi_{A, \varepsilon}$ est définie par:

$$
\psi_{A, \mathrm{~B}}=\exp -[\mathscr{A}(|x|)]^{2+\varepsilon} \text {. }
$$

Soit $A(s)$ une fonction vérifiant $\mathrm{H}_{\mathrm{I}}$, soit $\Omega=\boldsymbol{R}$ et considérons l'équation aux dérivées partielles:

$$
L_{2} u \equiv D_{x}\left(\sqrt{A}, D_{x} u\right)-D_{\iota} \frac{u}{\sqrt{A}}=0
$$

On suppose que $\dot{A}$ vérifie certaines propriétés de telle sorte que $F, F_{1}, G, G_{1}, H, C_{1}$ vérifient $\mathrm{H}_{\mathrm{II}_{A}}$.

Nous cherchons la solution $u$ du problème III sous forme de série; formellement nous posons:

$$
u=\sum_{m=0}^{\infty} a_{m}(x) f^{(m)}(t)
$$

où $a_{m}$ sont des fonctions de la variable $x$ et $f^{(m)}(t)$ est la dérivée $m^{\text {ì̀me }}$ d'une fonction de $t$.

En identifiant, nous avons:

$$
\sum_{m} f^{(m)}(t) D_{x}\left(\sqrt{A(|x|)} D_{x} a_{m}(x)\right)-\frac{1}{\sqrt{A(|x|)}} \sum_{m} a_{m}(x) f^{(m+1)}(t)=0 .
$$

D'où $\sqrt{A} \cdot D_{x} a_{0}=k$

$$
D_{x}\left(\sqrt{A} D_{x} a_{m+1}\right)=\frac{1}{\sqrt{A}} \alpha_{m}
$$

Et si $\mathscr{B}(x)=\int_{0}^{x} \frac{d x}{\sqrt{A}}$, nous avons:

$$
\begin{aligned}
a_{0} & =k \mathscr{B}(x), \\
a_{m} & =\frac{k}{(2 m+1) !}[\mathscr{B}(x)]^{2 m+1} .
\end{aligned}
$$


Soit $f$ une fonction indéfiniment dérivable, non identiquement nulle qui s'annule ainsi que toutes ses dérivées pour $t=0$ et qui vérifie

$$
\left|f^{(m)}(t)\right| \leqslant M_{1} m^{(1+\delta) m} \quad(\delta \text { nombre strictement positif) }
$$

(De telles fonctions existent: voir [2].)

On a alors:

$$
\left|\alpha_{m} f^{(m)}\right| \leqslant M_{1} k \frac{m^{\delta m}[\mathscr{B}(x)]^{2 m+1}}{m !}
$$

En utilisant la formule de Stirling, on a:

$\left|a_{m} f^{(m)}\right| \leqslant M \mathscr{B}(x)\left(\frac{[\mathscr{B}(x)]^{2}}{m^{1-\delta}}\right)^{m} \quad M$ constante positive indépendante de $m$.

Donc $\sum_{m} \alpha_{m} f^{(m)}$ est une série uniformément convergente sur tout borné de $\boldsymbol{R} \times[0, T]$. On peut démontrèr facilement que les séries obtenues en dérivant terme à terme sont elles aussi uniformément convergentes sur tout borne de $\boldsymbol{R} \times[0, T]$, c'est-à-dire que $u=\sum_{m} \alpha_{m} f^{(m)}(t)$ appartient à $C^{1,2}(S)$. De plus, $u \leqslant N \mathscr{B}(x) \exp [\mathscr{B}(x)]^{2 /(1-\delta)}$ voir [2].

Donc pour $\delta$ assez petit vérifiant $2 /(1-\delta)<2+\varepsilon / 2$, on montre facilement que $u$ appartient à $K_{\psi_{A, e^{*}}}$

5. Remarque. Une partie das résultats de [3] s'obtient facilement en choisissant correctement la fonction $A$.

(1) Si $A(s)=(2+s)^{\lambda}, 0 \leqslant \lambda<2$, nous obtenons les résultats des théorèmes 2.1.1, 2.2.1, 3.1.1, 3.2.1, de [3].

(2) $\mathrm{Si} A(s)=(3+s)^{2}[\log (3+s)]^{\nu}, \quad 0 \leqslant v \leqslant 1$, nous obtenons les résultats des théorèmes $2.1 .2,2.2 .2,3.1 .2,3.2 .2$ de [3].

Nous citons (sans démonstration) les lemmes dont nous avons fait usage dans les théorèmes précédents

LEMME A. Soit $f$ une fonction définie dans $\bar{\Omega} \times\left[t_{1}, t_{2}\right]$, lipschitzienne sur tout borné de $\bar{\Omega} \times\left[t_{1}, t_{2}\right]$ et nulle sur $\Gamma \times\left[t_{1}, t_{2}\right]$. Alors nous avons, pour tout $t$ appartenant $\dot{a}\left[t_{1}, t_{2}\right]$

$$
\int_{\omega_{r}} D_{i} f d x=\int_{\sigma_{r}} f \frac{x_{i}}{r} d s .
$$

LEMME B. Soit $\Omega$ un ouvert de $\boldsymbol{R}^{n}$, soit $f$ une fonction appartenant à $C^{1}(\bar{\Omega})$ et nulle sur $\Gamma \times\left[t_{1}, t_{2}\right]$, soit $f_{+}(x)=\max (f(x), 0)$.

On a les résultats suivants:

(1) la fonction $f_{+}$admet presque partout dans $\Omega$ des dérivées partielles et si on appelle $\Omega_{1}$ l'ensemble des points $x$ de $\Omega$ tel que $f(x)>0$ et $\Omega_{2}$ 
l'ensemble des points $x$ de $\Omega$ où $f(x) \leqslant 0$, on a:

les restrictions à $\Omega_{1}$ de $D_{i} f_{+}$et $D_{i} f$ sont égales, la restriction à $\Omega_{2}$ de $D_{i} f_{+}$est nulle presque partout, (2) la fonction $f_{+}$est lipschitzienne sur tout borné. de $\bar{\Omega}$.

\section{Bibliographie}

[1] J. Chabrowski, Sur un système non linéaire d'inégalités différentielles paraboliques dans un domaine non borné, Ann. Polon. Math. 22 (1969), p. 27-35.

[2] I. M. Gelfand et G. E. Chilov, Les distributions, Collection Universitaire de Mathémetiques. Edit. Dunod.

[3] G. Reynaud, Quelques résultats sur les solutions de systèmes d'inéquations de type parabolique, Thèse-Univereité d'Aix-Marseille, No C.N.R.S.: A.0. 6791.

[4] - Notes aux C.R. Acad. Sci. Paris, 271, série A, 1970, p. 835-274, série A, 1972, p. 636-274, série A 1972, p. 777.

UNIVERSITE SILESIENNE, KATOWICE

U.E.R., MATHEMATIQUE - INFORMATIQUE

UNIVERSIT L DE MARSEILLE - LUMINY 